\title{
A new location for pediatric immunoglobulin G4 related disease: the biceps muscle
}

\author{
Semanur Özdel ${ }^{1 \oplus}$, Mesiha Ekim $^{1 \oplus}$, Gülşah Kaygusuz ${ }^{2 \oplus}$, Elif Çelikel ${ }^{1 \oplus}$, \\ Göksel Vatansever ${ }^{3 \oplus}$, Nurdan Taçyıldız ${ }^{4 \odot}$ \\ Divisions of ${ }^{1}$ Pediatric Rheumatology and ${ }^{4}$ Pediatric Hematology and Oncology, Department of Pediatrics, ${ }^{2}$ Department of Pathology, \\ Ankara University Faculty of Medicine, Ankara; ${ }^{3}$ Department of Pediatrics, Kırklareli Hospital, Kırklareli, Turkey.
}

\begin{abstract}
Background. Immunoglobulin G4 (IgG4)-related disease (IgG4-RD) is a systemic disorder of unknown etiology characterized by elevated serum IgG4 and tissue infiltration of IgG4-positive plasma cells. The disease was described in the pancreas, aorta, thyroid, salivary glands, periorbital tissues, kidneys, pericardium and lymph nodes.
\end{abstract}

Case. Here in, we report a first pediatric case report of IgG4-related disease who presented with a mass in skeletal muscle i.e., biceps muscle.

Conclusion. To the best of our knowledge, the involvement in skeletal muscle has previously not been reported in children.

Key words: immunoglobulin G4, immunoglobulin G4-related disease, biceps muscle.

Immunoglobulin G4 (IgG4)-related disease (IgG4-RD) is a systemic disorder of unknown etiology characterized by elevated serum IgG4 and tissue infiltration of IgG4-positive plasma cells. ${ }^{1}$ IgG4 positive plasma cell infiltrations were first recognized in the pancreas. Until 2003, these abnormalities were not viewed as a distinct condition..$^{2-5}$ Later on, the abnormalities related to the disease were described in other organs and tissues like aorta, thyroid, salivary glands, periorbital tissues, kidneys, pericardium and lymph nodes.,3 But the involvement in skeletal muscle has not been reported.

To the best of our knowledge, this is the first pediatric case report of IgG4-related disease who presented with a mass in skeletal muscle i.e., biceps muscle.

$\triangle$ Semanur Özdel

semanurozdel@gmail.com

Received 18th December 2018, revised 3rd February 2019, accepted 27th August 2019.

\section{Case Report}

A 14-year-old girl presented with swelling in the upper arm. Her physical examination indicated an approximately $6 \times 7 \mathrm{~cm}$ swelling in the right upper arm. Other system examinations were unremarkable. She had no other complaints. On admission, blood laboratory tests revealed that white blood cell count was $23500 / \mathrm{mm}^{3}$, hemoglobin $8,7 \mathrm{~g} / \mathrm{dl}$, platelets $878000 / \mathrm{mm}^{3}$, erythrocyte sedimentation rate was $130 \mathrm{~mm} / \mathrm{h}$, C-reactive protein 124,6 mg/dl (normal <20 $\mathrm{mg} / \mathrm{dl}$ ). Liver and renal function tests, muscle enzymes, C3 and C4 were normal. Anti-nuclear antibody and anti-neutrophil cytoplasmic antibodies were negative. Serum IgG4 concentration was $606 \mathrm{mg} / \mathrm{dl}$ (normal $<135 \mathrm{mg}$ / dl). Lung $x$-ray and abdominal ultrasonography were reported as normal. Contrast-enhanced magnetic resonance imaging of the right upper arm revealed a well-defined $62 \times 48 \times 50-\mathrm{mm}$ contrast-retained solid mass with septa in the biceps muscle in T1-weighted series (Fig. 1). Bone marrow aspiration was performed for possible malignancy exclusion, which was 


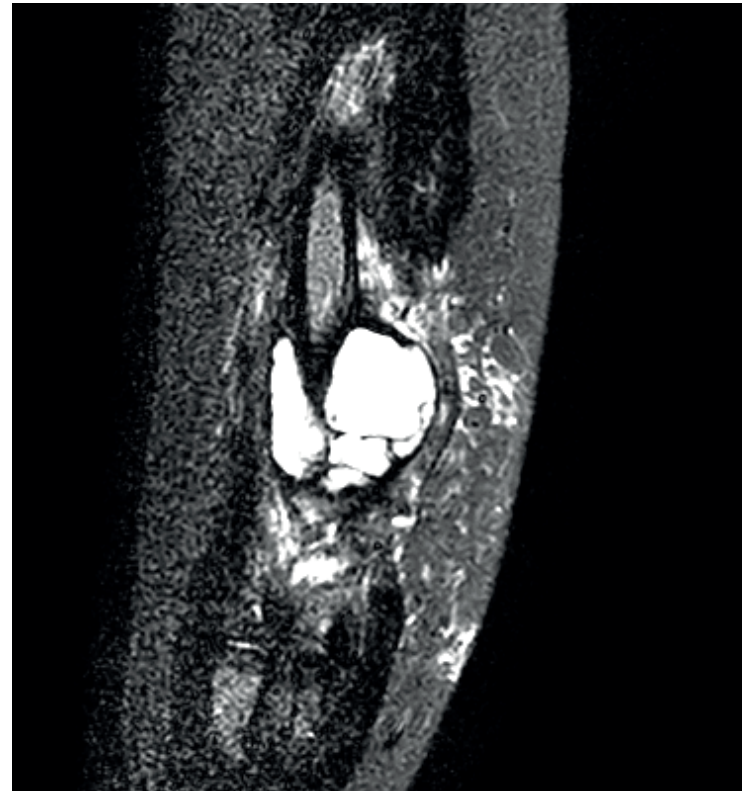

Fig. 1. Magnetic resonance imaging of right biceps muscle showed a well defined $62 \times 48 \times 50-\mathrm{mm}$ solid mass.

unremarkable. Then the mass was surgically dissected. The result biopsy of the mass showed intensive lymphoplasmocytic infiltration, enriched with IgG4-positive plasma cells (with an $\mathrm{IgG} 4 / \mathrm{IgG}$ ratio $>40 \%$ ), storiform fibrosis, and obliterative phlebitis (Fig. 2). There was no evidence of malignancy and granulomatous inflammation. In view of this, a diagnosis of 'IgG4-related disease' was rendered in this case. She was treated with prednisolone 2 milligram (mg) per kilogram (kg) per day per oral for a month. Then prednisolone therapy was slowly tapered. The mass shrunk to the level of being unpalpable. Acute phase reactants became within normal range. After follow up of 4 months, when prednisolone was tapered, her symptoms re-activated and the mass regrew. Then we started mycophenolate mofetil (MMF) $1200 \mathrm{mg}$ per square meters per day as an additional therapy to prednisolone but she responded poorly to the three-month MMF treatment. At follow up, three months later, rituximab was added to treatment and we observed a significant decrease the in the mass

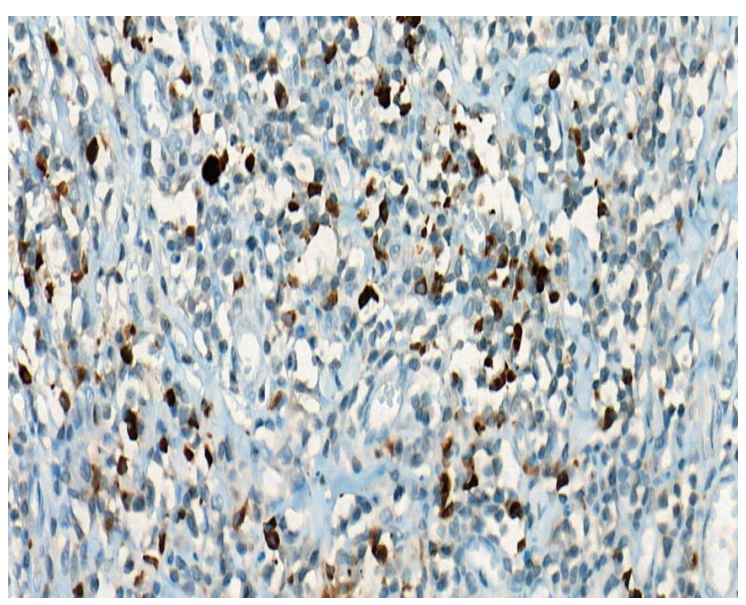

Fig. 2. IgG4 stain positive revealed an increased IgG4 plasma cell count.

volume (down to $2 \times 2 \mathrm{~cm}$ ) and prednisolone was stopped. She was successfully treated with rituximab for four months with resolution of symptoms.

Informed consent was received from the family.

\section{Discussion}

IgG4-RD is rare autoimmune disease condition. The exact etiology of IgG4-RD is unknown. Patients with IgG4-RD often present with tumor-like swelling. The three diagnostic criteria of Umehara are applicable to its diagnosis. ${ }^{1}$ A diagnosis of probable IgG4-RD requires the presence of a tumor-like swelling (clinical/radiological examination showing characteristic diffuse or localized swelling or masses in single or multiple organs) and one of the other criteria. The other criteria: Hematological examination showing elevated serum IgG4 concentrations (> $135 \mathrm{mg} / \mathrm{dl}$ ) and histopathological examination showing marked lymphocyte and plasmocyte infiltration, storiform fibrosis, and infiltration of IgG4+ plasma cells with a ratio of IgG4+/IgG+ plasma cells $\geq 40 \%$, and a total of $\geq 10$ IgG4+ plasma cells/ high power field (HPF). The presence of all three criteria indicates definite IgG4-RD. ${ }^{6,7}$ The majority of patients have high serumIgG4 
levels. But $30 \%$ of patients have normal serum IgG4 although typical histopathological and immunohistochemical findings. ${ }^{6}$ It should be noted that IgG4+ plasma cells may also be seen in diseases such as Castleman disease, eosinophilic granulomatosis with polyangiitis, sarcoidosis, inflammatory bowel disease and lymphoma but in these diseases, storiform fibrosis and obliterative phlebitis are not seen. This is especially important in the differential diagnosis. IgG4-RD can affect many organs and systems, commonly pancreas and other retroperitoneal organs. Various organ involvements have been described such as salivary glands, periorbital tissues, lymph nodes, biliary tree, kidneys, lungs, meninges, prostate, thyroid, pericardium, and skin. ${ }^{7.8}$ It is required for the differential diagnosis of many diseases in the diagnosis process. It is important to distinguish the disease from a variety of disorders, especially including neoplastic and inflammatory conditions.

Glucocorticoids are the most widely recommended agent at the first line of therapy. Usually the response is good. But the frequency of relapse is high with 25 to $50 \%{ }^{9,10}$ Besides, immunosuppressive therapy such as azathioprine or MMF are often require in many cases. In cases either recurrent or resistant disease, rituximab or bortezomib treatment is also recommended..$^{11,12}$

In conclusion, IgG4-RD is rare disease. The biceps muscle is a clinically rare region for IgG4RD. To the best of our knowledge this is the first pediatric case of IgG4-RD with upper arm muscle involvement. This case suggests that IgG4-RD can be consistent across a wide range of organ systems including skeletal muscles. If a patient presents with an tumoral formation in any organ and pathology cannot demonstrate malignancy, IgG4-RD should be considered.

\section{REFERENCES}

1. Umehara H, Okazaki K, Masaki Y, et al; Research Program for Intractable Disease by Ministry of Health, Labor and Welfare (MHLW) Japan G4 team. A novel clinical entity, IgG4-related disease (IgG4RD): general concept and details. Mod Rheumatol 2012; 22: 1-14.

2. Stone $\mathrm{JH}$, Khosroshahi A, Hilgenberg A, Spooner A, Isselbacher EM, Stone JR. IgG4-related systemic disease and lymphoplasmocytic aortitis. Arthritis Rheum 2009; 60: 3139-3145.

3. Stone JH, Zen Y, Deshpande V. IgG4-related disease. N Engl J Med 2012; 366: 539-551.

4. Hamano H, Kawa S, Horiuchi A, et al. High serum IgG4 concentrations in patients with sclerosing pancreatitis. N Engl J Med 2001; 344: 732-738.

5. Kamisawa T, Funata N, Hayashi $\mathrm{Y}$, et al. A new clinicopathological entity of IgG4-related autoimmune disease. J Gastroenterol 2003; 38: 982984.

6. Sah RP, Chari ST. Serologic issues in IgG4-related systemic disease and autoimmune pancreatitis. Curr Opin Rheumatol 2011; 23: 108-113.

7. Stone JH, Khosroshahi A, Deshpande V, et al. Recommendations for the nomenclature of IgG4related disease and its individual organ system manifestations. Arthritis Rheum 2012; 64: 3061-3067.

8. Pace C, Ward S. A rare case of IgG4-related sclerosing disease of the maxillary sinus associated with bone destruction. J Oral Maxillofac Surg 2010; 68: 25912593.

9. Masaki Y, Kurose N, Umehara H. IgG4-related disease: a novel lymphoproliferative disorder discovered and established in Japan in the 21st century. J Clin Exp Hematop 2011; 51: 13-20.

10. Palazzo E, Palazzo C, Palazzo M. IgG4-related disease. Joint Bone Spine 2014; 81: 27-31.

11. Khosroshahi A, Carruthers MN, Deshpande V, Unizony S, Bloch DB, Stone JH. Rituximab for the treatment of IgG4-related disease: lessons from 10 consecutive patients. Medicine (Baltimore) 2012; 91 : 57-66.

12. Khan ML, Colby TV, Viggiano RW, Fonseca R. Treatment with bortezomib of a patient having hyper IgG4 disease. Clin Lymphoma Myeloma Leuk 2010; 10: 217-219. 